\title{
Rhomboid domain containing 1 promotes the growth of non-small cell lung cancer through the activation of EGFR and regulation of the BIK-mediated apoptosis
}

\author{
Zhe-Yuan XU' ${ }^{1}$, Xu LI ${ }^{1}$, Rui-Hong YAO ${ }^{1}$, Li-Min YANG ${ }^{1}$, Hao PENG ${ }^{2}$, Wen-Jun REN ${ }^{1}$, Hong LU ${ }^{1}$, Ping WANG ${ }^{1, *}$ \\ ${ }^{1}$ Department of Thoracic Surgery, The Second Affiliated Hospital of Kunming Medical University, Kunming, Yunnan, China; ${ }^{2}$ Department of \\ Thoracic Surgery, First People's Hospital of Yunnan Province, Kunming, Yunnan, China \\ *Correspondence: pingwang2467@163.com; wangping2467@aliyun.com
}

Received August 4, 2021 / Accepted November 8, 2021

\begin{abstract}
RHBDD1 overexpression is found in various malignancies, including non-small cell lung cancer (NSCLC), and it is correlated with NSCLC patients' poor overall survival. This study aims to explore the function of RHBDD1 in regulating the progression of NSCLC and its potential molecular basis. qPCR, immunohistochemistry, and/or western blotting were used to evaluate the expression of RHBDD1 in NSCLC tissues and cell lines. RHBDD1 knockdown and overexpression were performed, CCK- 8 assay and cell clone formation were applied to study the function of RHBDD1 in cell proliferation in vitro. Flow cytometry and immunofluorescence tests were employed to determine the regulation of apoptosis, cell cycle, and endoplasmic reticulum stress by RHBDD1. As a result, RHBDD1 was found significantly upregulated in NSCLC tissues and cells and associated with pathological tumor staging. RHBDD1 knockdown inhibited the proliferation of NSCLC cells both in vitro and in vivo, promoted their apoptosis, caused cell cycle arrest at G0/G1 phase, characterized with reduced CDK2, suppressed TGF- $\alpha$ secretion, and inhibited the EGFR/Raf/MEK/ERK signaling pathway. In contrast, RHBDD1 overexpression showed the opposite effects. These effects of the manipulated expression of RHBDD1 on NSCLC were restored by EGFR or MEK inhibitor. Additionally, RHBDD1 knockdown and overexpression resulted in decreased and increased BIK cleavage, respectively, but the effects could be blocked by a proteasome inhibitor. In conclusion, our research shows that RHBDD1 promotes the progression of NSCLC through enhancement of proliferation and induction of apoptosis by regulating the EGFR/Raf/MEK/ERK signaling pathway and the level of BIK protein level.
\end{abstract}

Key words: RHBDD1, non-small cell lung cancer, TGF- $\alpha$, BIK, EGFR/Raf/MEK/ERK

Lung cancer is the most common cause of cancer-related death worldwide [1]. Non-small cell lung cancer (NSCLC) accounts for more than $80-85 \%$ of all lung cancers [2]. Among patients diagnosed with lung cancer, the 5-year survival rate is very low due to the advanced clinical manifestations and few targeted therapies [3, 4]. Although the detection, surgical, and chemotherapy drugs development have significantly improved the treatment of lung cancer, the 5 -year-survival rate remains poor. Therefore, it is very necessary to explore further molecular pathogenesis of NSCLC and potential therapeutic targets for its treatment.

The rhomboid protease is a very conserved serine protease family that regulates many important cellular functions [5]. Among them, RHBDD1 is a mammalian serine protease in the membrane [6]. RHBDD1 promotes tumor metastasis through the Wnt signaling pathway and ZEB1 in metastatic colorectal cancer [7]. The highly up-expressed RHBDD1 in breast cancer promotes the proliferation and invasion ability by regulating the Akt signaling pathway and the expression of CDK2. RHBDD1 is linked to the progression of pathological breast cancer stages [8]. Mammalian rhomboid protease RHBDD1 promotes secretion of the EGFR ligand, transforming growth factor $\alpha$ (TGF- $\alpha$ ) [9]. The high expression of RHBDD1 in colon cancer induces the ADAM-independent cleavage and secretion of proTGF- $\alpha$. The secreted proTGF- $\alpha$ matures and further triggers the EGFR/Raf/MEK/ERK signaling pathway, and ultimately promotes the growth of colon cancer cells [10]. Overexpression of RHBDD1 in lung cancer tissues is associated with a low survival rate of lung cancer patients. Downregulation of RHBDD1 can inhibit the proliferation, migration, and invasion of lung cancer cells [11]. However, there are few reports on the mechanism 
of RHBDD1 regulating the proliferation and apoptosis of NSCLC cells.

Previous studies have shown that TGF- $\alpha$ was an important factor in the abnormal proliferation of malignant tumors and could be activated by mammalian TNF- $\alpha$ converting enzyme (TACE) [12]. The activated form of TGF- $\alpha$ further binds to EGFR (EGF receptor) to activate the EGFR signaling pathway [13]. Overexpression of TGF- $\alpha$ is carcinogenic, which not only promotes the malignant proliferation of transgenic mice tumors but also promotes the higher tumorigenicity of human cancer cells in ectopically expressed nude mice [14-16]. Overexpression of TGF- $\alpha$ can lead to pancreatic fibrosis and further pancreatic ductal carcinoma [17]. However, whether there is a regulatory link between RHBDD1 and TGF- $\alpha$ has not yet been reported.

Apoptosis promotes tumor cell death, and exploring the molecular mechanism of tumor cell apoptosis is beneficial for cancer treatment [18]. A low level of apoptosis or inhibition of apoptosis is one of the main characteristics of tumors, which can lead to the progression of cancer [19]. The Bcl-2 family is a class of protein molecules that regulate the process of cell apoptosis. Bcl-2 interaction killer (BIK), a pro-apoptotic member of the Bcl-2 family, can induce apoptosis of mammalian cells under various stimuli and is associated with recurrence of clinical breast cancer as a potential therapeutic target [20]. The activation of BIK is tightly regulated by RHBDD1 in human testicular cells, resulting in reduced BIK-mediated apoptosis [21]. In addition, RHBDD1, as an anti-apoptotic protein, inhibits cell apoptosis by upregulating the activity and expression of AP-1 and Bcl-2 [22]. In general, the ability of RHBDD1 for regulating apoptosis makes it a potential target for cancer treatment. However, whether RHBDD1 exacts anti-apoptotic ability in NSCLC and the underlying molecular mechanism remains unclear.

In this study, through various molecular and cellular experiments, we mainly demonstrated that overexpressed RHBDD1 in NSCLC promotes the proliferation of NSCLC cells both in vitro and in vivo, inhibits their apoptosis, in which, activated EGFR/Raf/MEK/ERK signaling pathway participated, in addition, increased BIK cleavage also played a role. Therefore, it is concluded that RHBDD1 promotes the progression of NSCLC through enhancement of proliferation and induction of apoptosis by regulating the EGFR/Raf/MEK/ERK signaling pathway and the level of BIK protein level.

\section{Materials and methods}

Immunohistochemistry. The tumor tissues and corresponding adjacent normal tissues of 45 patients with NSCLC from our hospital were subjected to immunostaining scores to evaluate protein expression levels. The patient consent and approval from the institutional research ethics committee have been obtained prior to the use of these patient specimens for research purposes. The study complies with the ethical guidelines of the Declaration of Helsinki.

First, the embedded samples were cut into approximately $4 \mu \mathrm{m}$ thick sections, deparaffinized in xylene, and dehydrated in graded alcohols of different concentrations. Then, the sections were treated in $10 \mathrm{mM}$ citrate buffer $(\mathrm{pH} 6.0)$ at $95^{\circ} \mathrm{C}$ for $15 \mathrm{~min}$ for antigen retrieval, and then endogenous peroxidase was blocked with $3 \%$ hydrogen peroxide for 10 min. Next, the sections were incubated overnight at $4{ }^{\circ} \mathrm{C}$ with the primary antibody against RHBDD1 (PA5-110291, Thermo Fisher, USA), and washed with phosphate-buffered saline (PBS) and incubated with HRP-conjugated secondary antibody (ab288151, Abcam) at $37^{\circ} \mathrm{C}$ for $30 \mathrm{~min}$.

Cell culture and reagents. Human NSCLC cell lines A549, H1299, H1550, H1975, SPCA-1, SK-MES, and normal lung cell line $(\mathrm{HBE})$ were purchased from the National Collection of Authenticated Cell Cultures (Shanghai, China) and cultured in RPMI-1640 medium containing 10\% fetal bovine serum. All cell lines were incubated in a humidified atmosphere containing $5 \% \mathrm{CO}_{2}$ at $37^{\circ} \mathrm{C}$. The EGFR kinase inhibitor AG1478 was purchased from Selleck Chemicals (Houston, TX, USA), and the ERK1/2 inhibitor U0126 was purchased from Cell Signaling Technology (Beverly, MA, USA).

siRNA synthesis, plasmid construction, and cell transfection. According to the previous research reports, the sequence of RHBDD1 siRNA (siRHBDD1) and negativecontrol siRNA (si-NC) was referred to the former research and synthesized by Sangon Biotech [23]. The cDNA of RHBDD1 were amplified and then cloned to the pcDNA3.1 vector. The empty vector pcDNA3.1 was used as a negative control plasmid. The plasmids were transfected to the A549 and H1299 cells with Lipo3000 (Invitrogen, Carlsbad, CA, USA), respectively. The expression level of RHBDD1 was analyzed with qPCR and western blot analysis after $48 \mathrm{~h}$. The level of soluble TGF- $\alpha$ in the cell supernatant was measured by enzyme-linked immunosorbent assay (ELISA) according to the manufactures' instructions (ab100646, Abcam).

Western blotting. The cells to be tested were dissolved in SDS lysis buffer to extract protein, and BCA reagent (Thermo Fisher) was used for protein quantification. The cell lysate was separated by $10 \%$ SDS-PAGE gel and then transferred to the NC membrane. The membrane was blocked in 5\% skim milk for $1 \mathrm{~h}$ at room temperature. After the blocking, the membrane was incubated in the designated antibody at $4{ }^{\circ} \mathrm{C}$ overnight. Finally, the membrane was incubated with antirabbit or anti-mouse HRP-IgG secondary antibody at room temperature for $1 \mathrm{~h}$. ECL imprinting detection system was used to detect chemiluminescence. Antibodies against BIK or cleaved-BIK (3703-100) was purchased from Biovision. Antibody against RHBDD1 (PA5-110291) was from Thermo Fisher. Antibodies against CDK2 (\#2546), CDK4 (\#12790), Cyclin D1 (\#2978), phospho-c-Raf (\#9421), c-Raf (\#53745), phospho-MEK1/2 (\#3958), MEK1/2 (\#4694), phosphoERK1/2 (\#9521), ERK1/2 (\#5013), phospho-EGFR (\#3777), or EGFR (\#4267) were purchased from Cell Signaling 
Technology, and antibody against p-Akt ${ }^{\mathrm{Ser} 433}$ (ab81283), cleaved-caspase 3 (ab184787), cleaved-caspase 7 (ab256469) or $\beta$-actin (ab8227) were from Abcam (Cambridge, UK).

Cell viability assay. The CCK- 8 method was used to determine and assess the level of cell proliferation. The cells were cultured in 96-well plates at the density of 3,000 cells/well, and a fresh medium was replaced every three days. After the incubation was over, $100 \mu \mathrm{l}$ fresh medium and $10 \mu \mathrm{l} \mathrm{CCK-8}$ solvent was added to each well and cultured at $37^{\circ} \mathrm{C}$ for $2 \mathrm{~h}$. The absorbance at $450 \mathrm{~nm}$ was measured using a microplate reader.

Colony formation assay. 1,000 cells/well were inoculated in a 6-well plate. The cells were cultured for 14 days until the cell colonies were visible. Cells were then stained with $0.1 \%$ crystal violet and photographed, and the number of communities was calculated.

Cell cycle and apoptosis assay. The cells were treated simultaneously with double thymidine (TdR) in the G1/S phase, and the operation was carried out in accordance with the method reported in the study [24]. A549 cells were blocked with $2 \mathrm{mM}$ TdR for $16 \mathrm{~h}$, then released in the fresh medium for $12 \mathrm{~h}$ (for A549 cells and RHBDD1-OE (RHBDD1 overexpression) A549 cells) or $14 \mathrm{~h}$ (for RHBDD1-KD (RHBDD1 knockdown) A549 cells), and then further incubated in 2 mM TdR for 18 h. H1299 cells were blocked with 2 mM TdR for $17 \mathrm{~h}$, then released in the fresh medium for $9 \mathrm{~h}$ (for $\mathrm{H} 1299$ cells and RHBDD1-OE H1299 cells) or 12 h (for RHBDD1KD H1299 cells), and then further incubated with $2 \mathrm{mM}$ TdR for $17 \mathrm{~h}$. After washing twice with PBS, the cells were resuspended in a fresh medium.

Following the reagent manufacturer's instructions, the cells were stained with propidium iodide (PI; Millipore). CellQuest software (BD Biosciences, CA, USA) was used to analyze the results. The cells were stained with $5 \mu$ AnnexinV-FITC and $10 \mu \mathrm{l}$ PI Staining Solution (Sigma, St. Louis, MO, USA) according to the reagent manufacturer's instructions. The proportion of apoptotic cells was analyzed by the flow cytometric analysis.

In vivo tumorigenesis. Five million tumor cells in good condition were selected and suspended in $200 \mu \mathrm{l}$ of PBS and injected into the flank of 6-week-old athymic glands in each group $(n=5)$. Every three days, the mouse tumor growth was detected and measured, and the tumor width and length were recorded. The mice were sacrificed 20 days after the inoculation, and the tumor tissue was submitted to IHC (immunohistochemistry) analysis to detect the level of proliferation biomarkers (Ki67 \& PCNA).

Immunofluorescence. Cells in different groups were fixed with $4 \%$ PFA at $4{ }^{\circ} \mathrm{C}$, permeabilized with $0.3 \%$ Triton X-100 in PBS, and blocked with $10 \%$ serum. Then use anti-human K48-Ub antibody (ab140601, Abcam) to incubate overnight at $4{ }^{\circ} \mathrm{C}$. Then the cells were stained with Alexa Fluor ${ }^{\circledR} 594$ conjugated goat anti-rabbit IgG secondary antibody (red; A-11012, Thermo Fisher) and counterstained with DAPI (blue).
Quantitative real-time PCR (qRT-PCR). TRIzol reagent (Life Technologies, USA) was used to isolate total RNA from cells and tissues. The PrimeScript RT reagent Kit (Takara, Japan) and the SYBR PrimeScript RT-PCR Kit were used to generate complementary DNA of mRNA. The expression of RHBDD1 was measured using SYBR Green Master Mix (Takara, Japan), and ACTB was used as the internal control. The PCR primers were as follows: RHBDD1, forward, 5'-ATGCAACGGAGATCAAGAGGG-3' and reverse, 5'-GCAGGAGCTATACAGTGGCTTC-3'; ACTB, forward, 5'-CCATGTACGTTGCTATCCAGG-3' and reverse, 5'-TCTCCTTAATGTCACGCACGA-3'. Quantitative analysis was calculated using the $2^{-\Delta \Delta \mathrm{Ct}}$ method.

Statistical analysis. In this study, graphs were produced by GraphPad Software V8.0. Student's t-test was used to compare the two groups, and for multiple comparisons (more than two groups), ANOVA analysis was employed to show the significance, and Tukey test was employed as Post Hoc test for multiple comparisons of groups. The experimental data were expressed as mean \pm SD. A p-value $<0.05$ was considered statistically significant.

\section{Results}

RHBDD1 is highly expressed in NSCLC clinical tissues and cell lines. At the beginning of this research, we noticed that the expression level of RHBDD1 is elevated in several types of cancers, and validated it in lung adenocarcinoma (LUAD) and lung squamous cell carcinoma (LUSC) clinical samples from TCGA (The Cancer Genome Atlas) database (https://portal.gdc.cancer.gov/) using the online tool GEPIA2 (http://gepia2.cancer-pku.cn/). To confirm the potential role of RHBDD1 in the development and progression of NSCLC, we analyzed RHBDD1 expression in 45 paired NSCLC tissues and adjacent tissues by RT-PCR and immunohistochemistry (IHC). As shown in Figure 1A, the expression of RHBDD1 was significantly increased in NSCLC tissues compared to adjacent tissues. The median expression in NSCLC tumors was 3.5 times that of normal tissues. The results of subsequent IHC experiments further confirmed this conclusion (Figure 1B). Also, we noticed that RHBDD1 expression was localized in the plasma membrane and cytoplasm. With the increase of $\mathrm{pT}$ (pathological tumor) staging, the expression of RHBDD1 in NSCLC tissues was generally gradually and seriously increased (Figure 1B). At the same time, RHBDD1 expression was detected in several NSCLC cell lines. As shown in Figures 1C and 1D, RHBDD1 was generally highly expressed in NSCLC cell lines compared to normal lung bronchial epithelial cell line HBE. Among them, RHBDD1 protein was the most significantly higher in A549 and H1299 cells.

RHBDD1 is positively correlated with the proliferation of NSCLC cells. In order to explore the regulatory role of RHBDD1 in NSCLC cells in vitro, we selected A549 and H1299 cell lines with high RHBDD1 expression. The expres- 
A

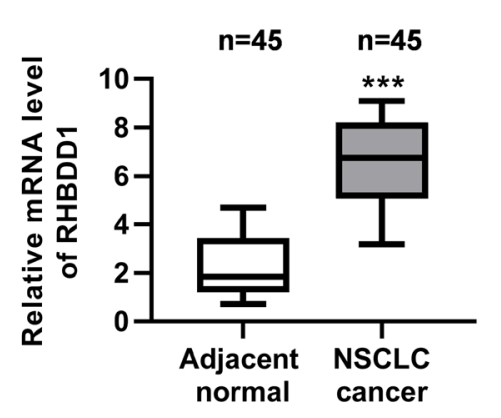

C

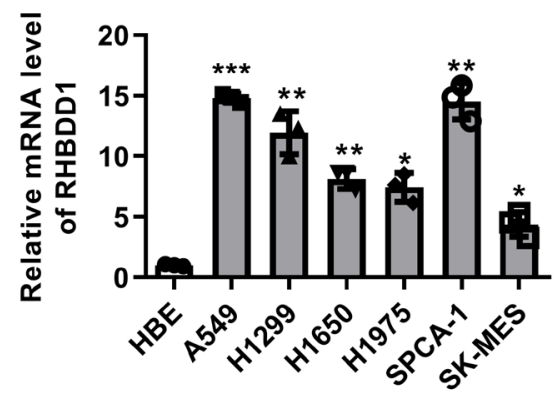

B
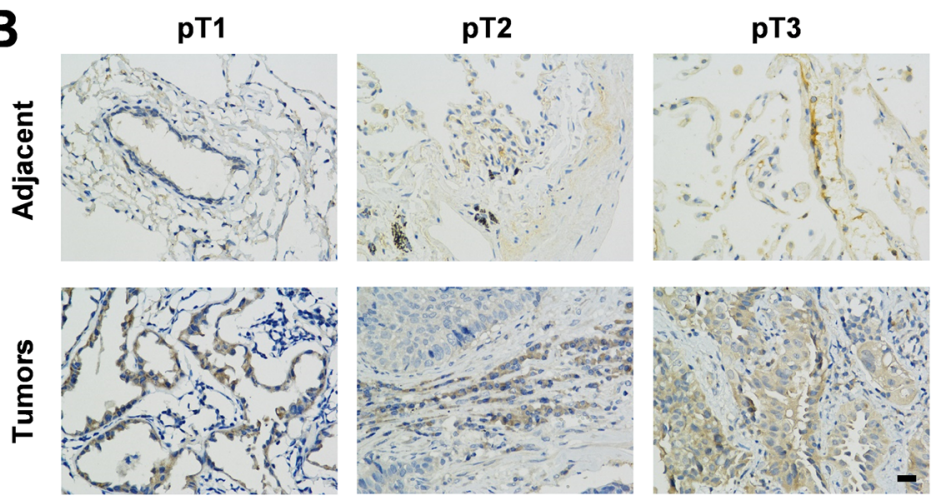

D

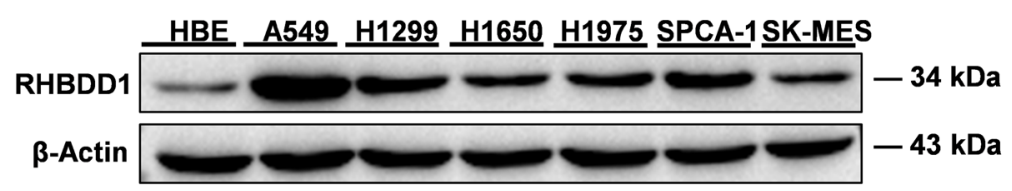

Figure 1. RHBDD1 expression is generally high in NSCLC tissues and cells. A) RT-PCR analysis was employed to detect the expression level of RHBDD1 in adjacent normal tissues $(n=45)$ and NSCLC tissues $(n=45) .{ }^{* *} p<0.001$ vs. adjacent normal. B) Immunohistochemical $($ IHC) analysis of the RHBDD1 expression between the different phases of NSCLC tissues and the adjacent tissues. Scale bar $200 \mu \mathrm{m}$. C) RT-PCR analysis was employed to detect the expression level of RHBDD1 in human bronchial epithelial cells and the NSCLC cell lines. ${ }^{\star} \mathrm{p}<0.05,{ }^{* *} \mathrm{p}<0.01,{ }^{* * *} \mathrm{p}<0.001$ vs. HBE cells. D) Western blot analysis was employed to detect the expression level of RHBDD1 in human bronchial epithelial cells and the NSCLC cell lines. ${ }^{\star \star}$ p $<0.01$, ${ }^{* * *} \mathbf{p}<0.001$ vs. HBE cell.

sion of RHBDD1 was knocked down by specific siRNA targeting at RHBDD1. Cell proliferation levels of A549 and H1299 were significantly reduced after RHBDD1 knockdown (Figure 2A). Subsequent analysis of clone formation further demonstrated that colony number was remarkably lower in RHBDD knockdown cells than in normal A549 and H1299 (Figure 2B). In contrast, after transfection of RHBDD1 expression plasmid in A549 and H1299 cells, the proliferation ability was elevated in A549 and H1299 cells. Next, we analyzed the effect of RHBDD1 on the proliferation cycle of NSCLC cells. Cell cycle flow analysis showed that RHBDD1 knockdown significantly decreased the proportion of the S phase cells in both A549 and H1299 cells, while its overexpression significantly increased the number of the cells at the S phase. The findings in H1299 cells were consis- tent with those in A549 cells. On the contrary, overexpression of RHBDD1 in A549 and H1299 cells showed a reverse signature of the cell cycle (Figures 2C, 2D).

We further investigated whether RHBDD1 changed the signal pathways related to the cell cycle and G1/S phase transition, thereby affecting the cell cycle progression and the G1/S phase arrest. Western blotting results showed that RHBDD1 knockdown inhibited the expression levels of cyclin-related proteins CDK2 and p-Akt phosphorylation at Ser473 (Figure 2E). Conversely, further upregulation of RHBDD1 expression partially promoted the levels of these molecules (Figure 2E). RHBDD1 knockdown also inhibited tumor growth in vivo in the subcutaneous tumor-bearing assay, while RHBDD1 overexpression promoted tumor growth (Figures 3A, 3B). Immunohistochemical experi- 
A

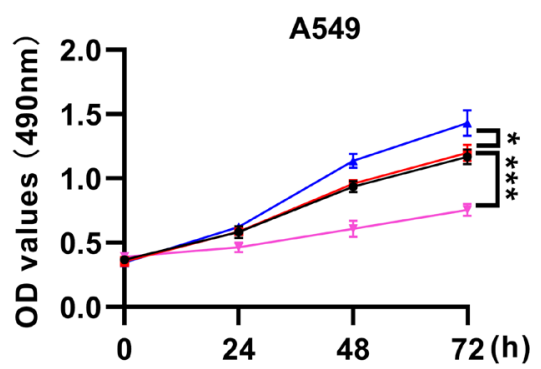

H1299

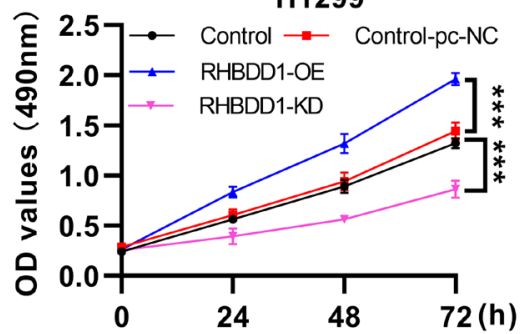

C
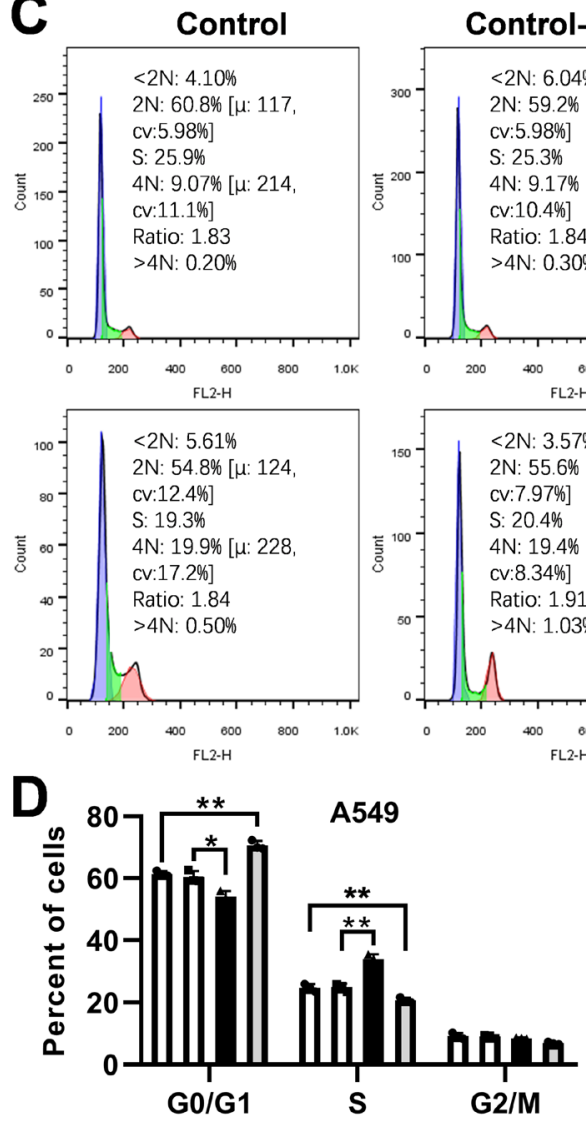

Control-pc-NC
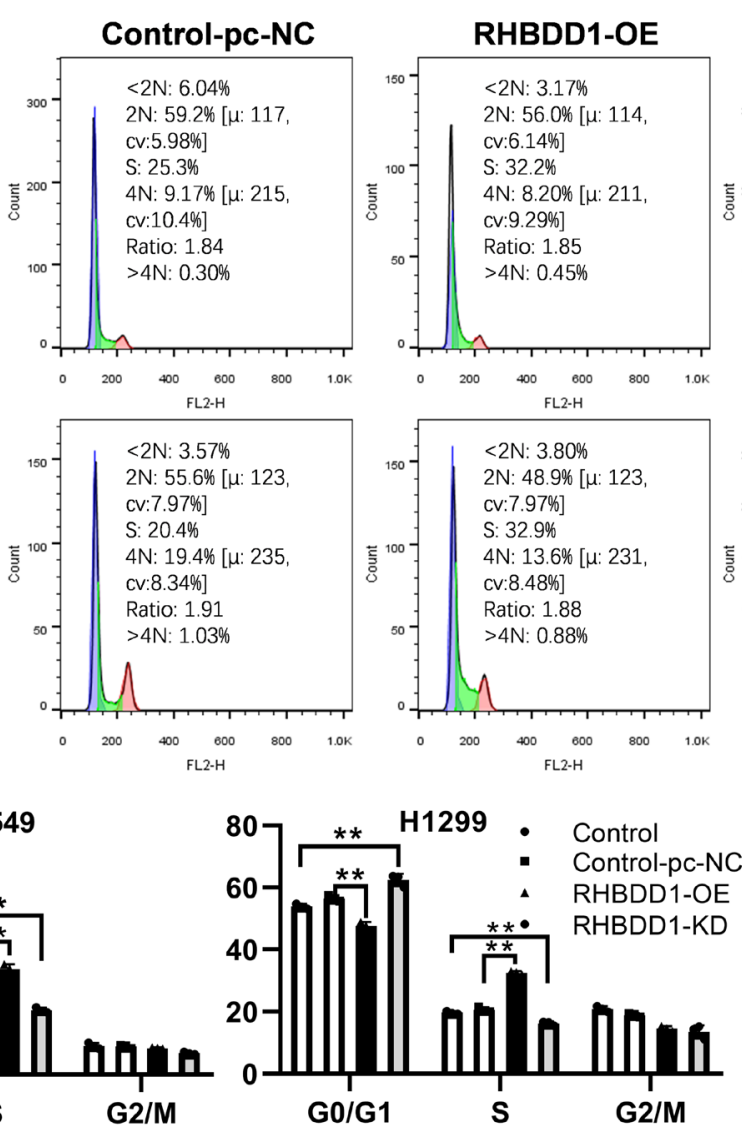

RHBDD1-OE RHBDD1-KD
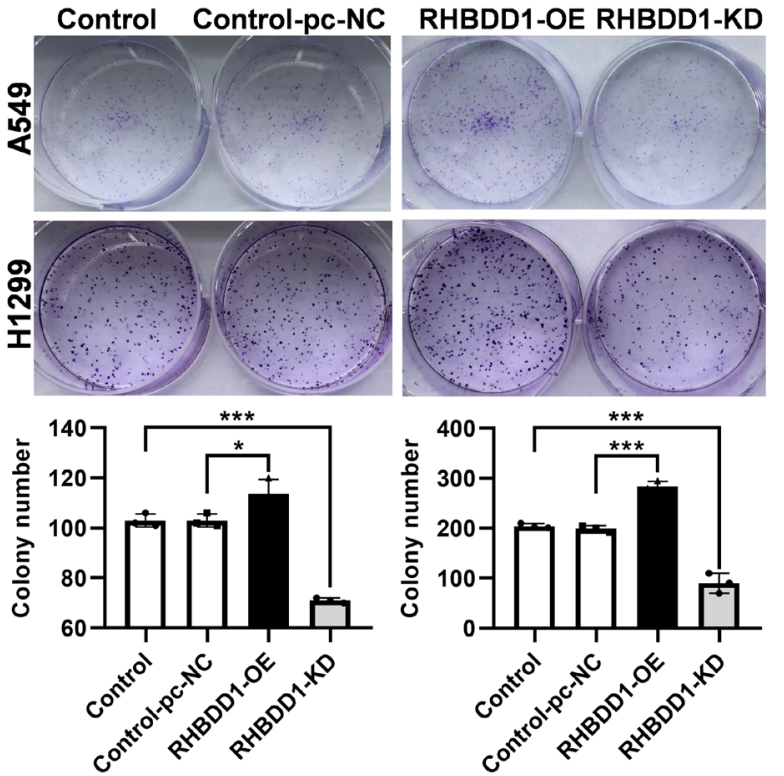
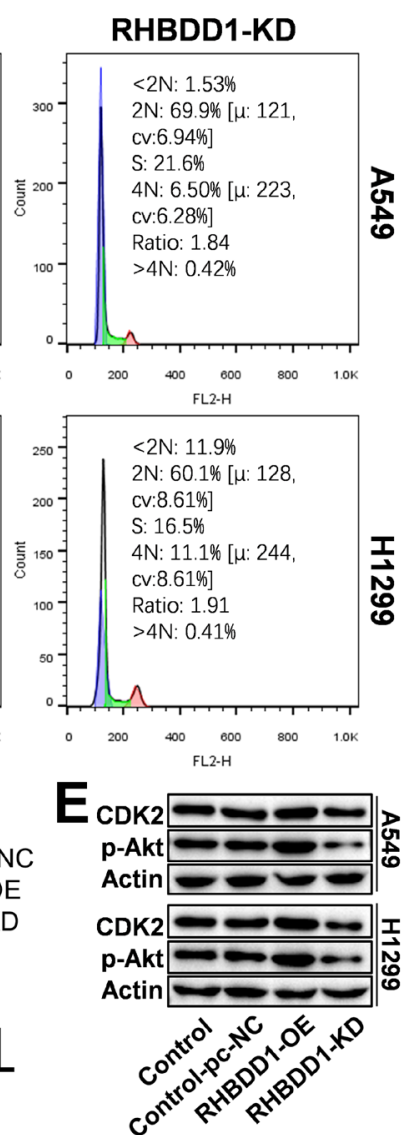

Figure 2. RHBDD1 is positively correlated with the proliferation of NSCLC cells in vitro. A) A CCK-8 assay was employed to analyze the effects of up- or downregulation of RHBDD1 on cell viability in NSCLC cell lines A549 and H1299. ${ }^{\star} p<0.05,{ }^{* * *} p<0.001$ vs. control or control-pc-NC. B) Cell clone formation assay was employed to analyze the effects of up- or downregulation of RHBDD1 on cell proliferation in NSCLC cell lines A549 and H1299. ${ }^{\star} p<0.05,{ }^{* * *} p<0.001$ vs. control or control-pc-NC. C, D) Flow cytometry analysis was employed to analyze the effect of up- and downregulated RHBDD1 on the cell cycle in A549 and $\mathrm{H} 1299$ cell lines. ${ }^{\star} \mathrm{p}<0.05,{ }^{* * *} \mathrm{p}<0.001$ vs. control or control-pc-NC. E) Western blot analysis was employed to analyze the regulatory role of RHBDD1 on CDK2 and p-Akt. 
A

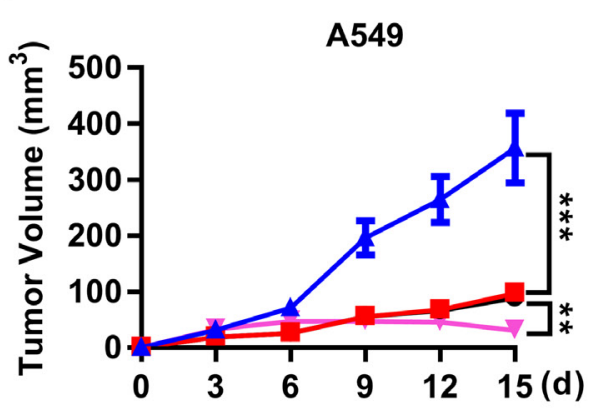

H1299

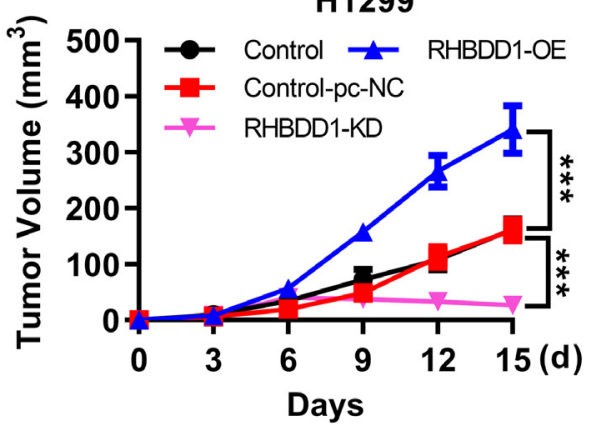

B

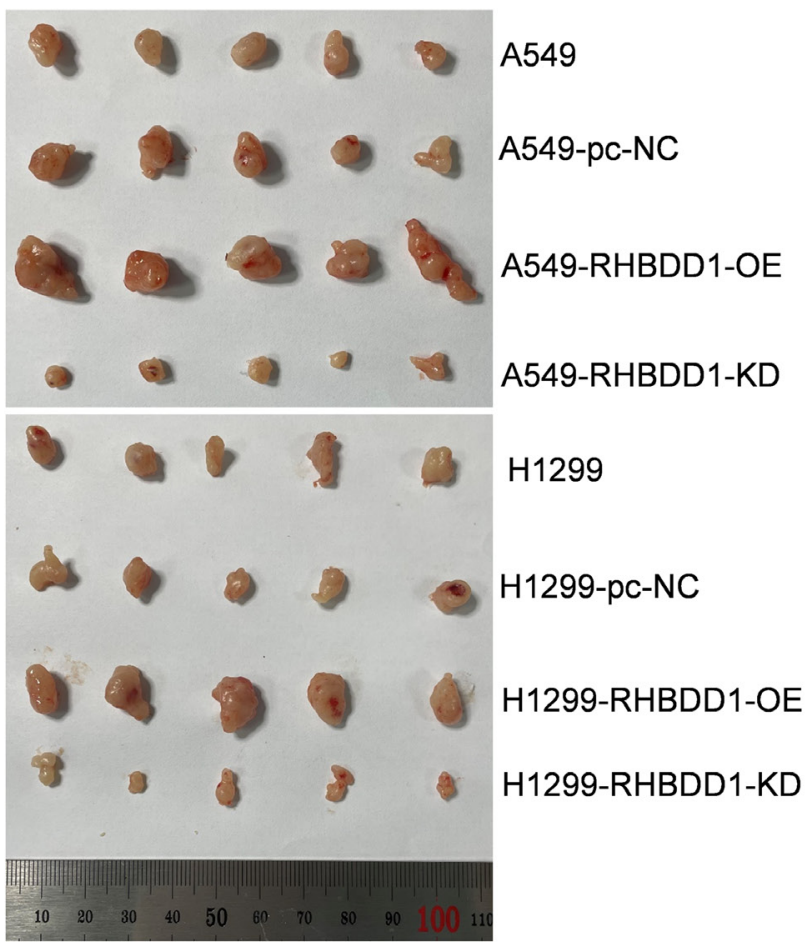

C

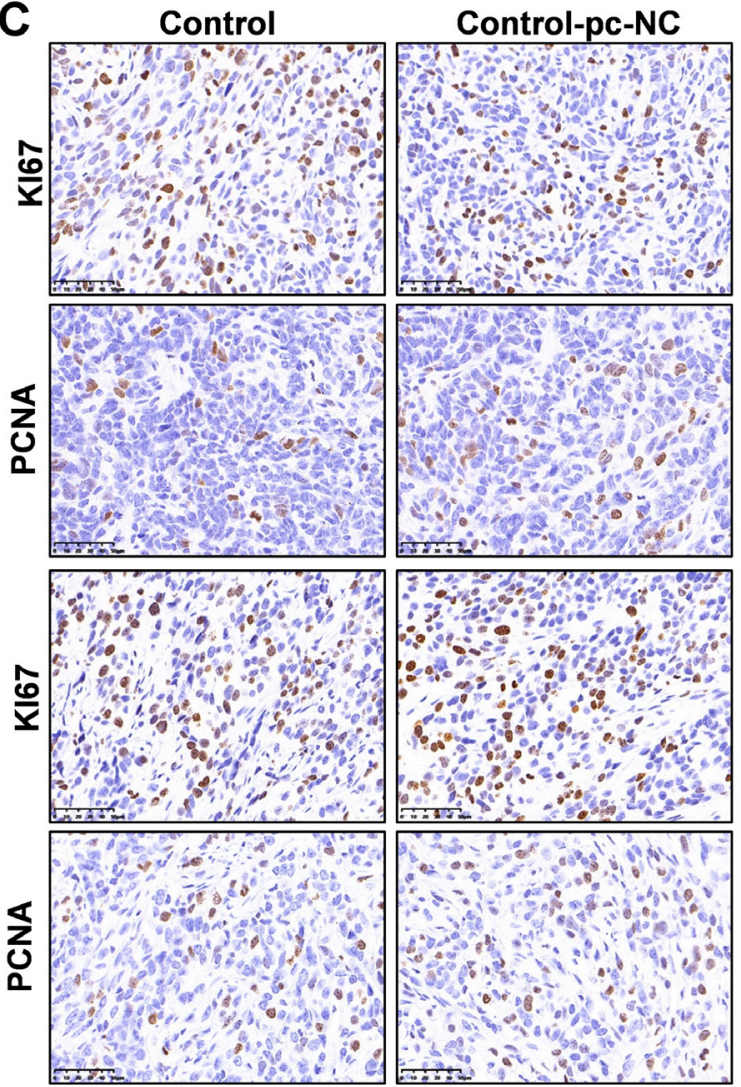

RHBDD1-OE
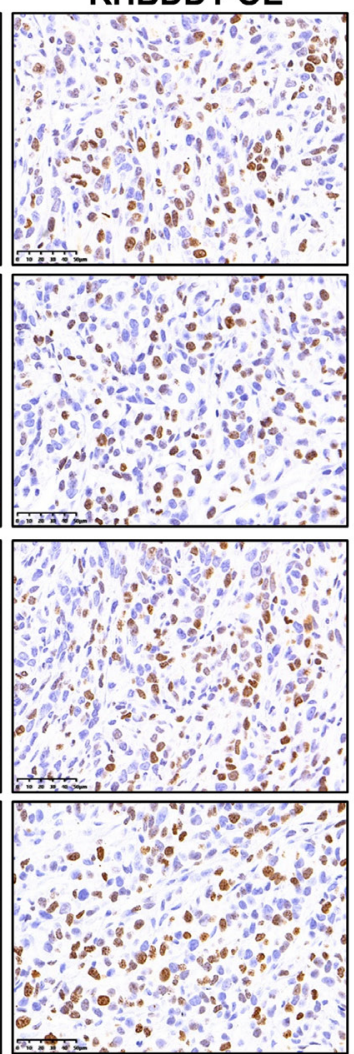

RHBDD1-KD

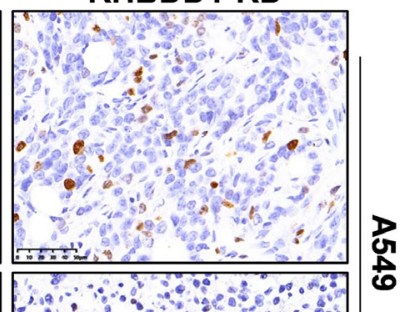

Figure 3. RHBDD1 is positively correlated with the growth of NSCLC cells in vitro. A, B) The tumor volume of each group was calculated and stripped out for macroscopy after execution. ${ }^{* *}$ p $<0.001$ vs. control or control-pc-NC groups. C) IHC analysis of Ki67 and PCNA expression of the xenografts tissue. Scale bar $50 \mu \mathrm{m}$ 
ments showed that, at the molecular level, RHBDD1 knockdown reduced the expression of Ki67 and PCNA, markers of cell proliferation (Figure 3C).

RHBDD1 induces the secretion of TGF- $\alpha$ and then activates the EGFR/Raf/ERK signaling pathway to promote cell proliferation. Previous studies have shown that RHBDD1 can promote the expression of TGF- $\alpha$ in colon cancer, and then activate the EGFR/Raf/ERK signaling pathway. We analyzed whether RHBDD1 has a similar promoting function in NSCLC. The enzyme-linked immunosorbent assay results showed that the overexpression of RHBDD1 in A549 cells further stimulated the secretion of TGF- $\alpha$. The knockdown of RHBDD1 significantly reduced the expression of TGF- $\alpha$ in the supernatant of cells, but the following overexpression of RHBDD1 can rescue this process (Figure 4A). As an activator of the EGFR receptor, TGF- $\alpha$ secreted by cells promotes the phosphorylation and activation of EGFR. Our results showed that RHBDD1 overexpression induced increased secretion of TGF- $\alpha$ promoted the levels of p-EGFR, p-Raf, and p-ERK (Figure 4B). Conversely, RHBDD1 knockdown inhibited activation of the EGFR/Raf/ ERK signaling pathway (Figure 4B).
We examined whether RHBDD1 has the promotive regulatory effect on NSCLC cell proliferation through the EGFR/Raf/ERK signaling. The results shown in Figure 4C indicated that the overexpression of RHBDD1 could significantly promote cell proliferation. However, the addition of the EGFR inhibitor AG1478 almost completely inhibited the proliferation process of cancer cells, while MEK inhibitor U0126 partially inhibited the proliferation number of cancer cells. Similar results were found in the following clone formation experiment (Figure 4D). These results suggest that RHBDD1 promotes the proliferation of cancer cells through the EGFR/RAF/ERK signaling pathway in NSCLC.

RHBDD1 inhibits apoptosis by modifying and cutting downstream BIK. FACS analysis showed that the percentage of total apoptotic cells in A549 cells significantly increased in RHBDD1-KD cells (Figures 5A, 5B). Conversely, RHBDD1-overexpression (OE) partially decreased apoptosis rates in A549 and H1299 (Figures 5A, 5B). At a molecular level, RHBDD1 knockdown promoted cleavage of caspase 3 and caspase 7 expression in A549 and H1299 cells. On the contrary, overexpression of RHBDD1 further inhibited the cleavage (Figure 5C). In terms of the
A

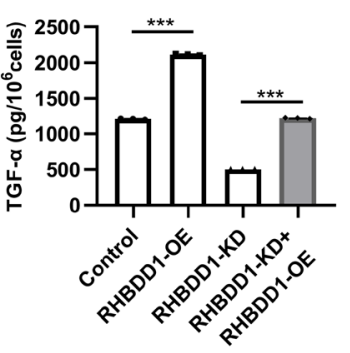

D

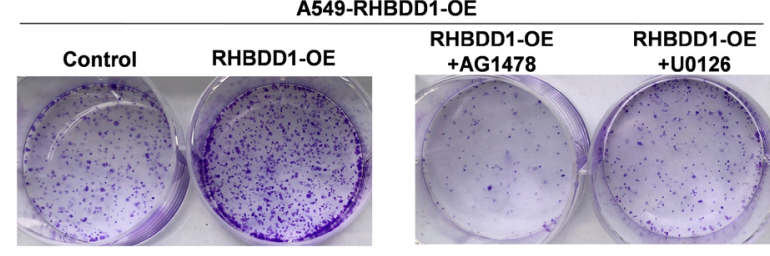

H1299-RHBDD1-OE
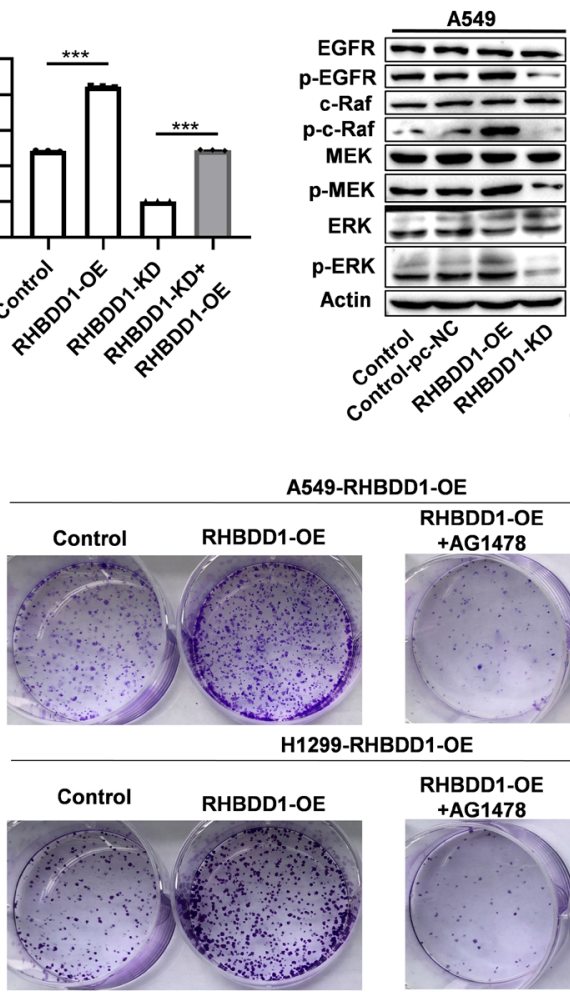

C
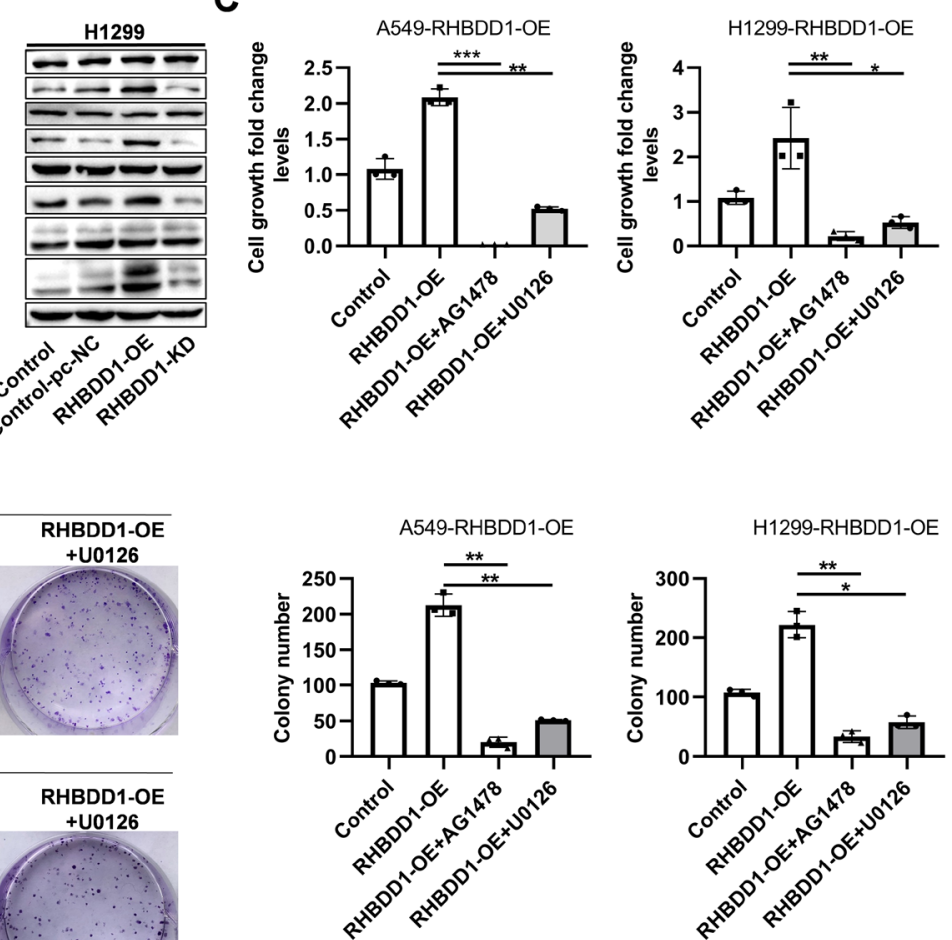

Figure 4. RHBDD1 regulates the EGFR/Raf/MEK/ERK signaling pathway to promote cell proliferation. A) The level of soluble TGF- $\alpha$ in the supernatant of each group of cells was measured by enzyme-linked immunosorbent assay (ELISA). ${ }^{\star * *}$ p $<0.001$. B) Western blotting was applied to analyze the effect of up- or downregulation of RHBDD1 on the activity of the EGFR/Raf/MEK/ERK signaling pathway. C) CCK-8 analyses were employed to analyze the cell viability ability of each group. ${ }^{\star} \mathrm{p}<0.05,{ }^{\star *} \mathrm{p}<0.01,{ }^{* * *} \mathrm{p}<0.001 \mathrm{vs}$. RHBDD1-OE group. D) Clone formation analyses were used to determine the cell proliferation ability of cells in each group. ${ }^{\star} \mathrm{p}<0.05,{ }^{* *} \mathrm{p}<0.01,{ }^{* *} \mathrm{p}<0.001$ vs. RHBDD1-OE group. 
mechanism of RHBDD1 regulating apoptosis, we speculated that RHBDD1 inhibited the process of apoptosis by modifying and splicing downstream BIK. Through western blotting analysis (Figure 5D), we found that overexpression of RHBDD1 in A549 and H1299 cells increased cleaved BIK level, and RHBDD1 knockdown further decreased cleavedBIK level compared to control groups. However, the effects of the manipulated RHBDD1 expression could be blocked by the proteasome inhibitor Velcade (Figure 5D).

In addition, we found that the overexpression of RHBDD1 in H1299 cells promoted the accumulation of endogenous K48 polyubiquitinated protein. Conversely, knockdown of RHBDD1 further inhibited the expression of K48 polyubiquitinated protein (Supplementary Figure S1).

\section{Discussion}

A recent study has shown that RHBDD1 was highly expressed in colorectal cancer tissues and was closely related to the survival of colorectal cancer patients [10]. RHBDD1 in colorectal cancer promotes tumor proliferation and metastasis by activating the EGFR/Raf/MEK/ERK signaling pathway and ZEB1 gene. After the knockdown of RHBDD1 in vivo and in vitro, the proliferation ability and migration/ invasion ability of colorectal cancer cells decreased significantly $[7,10]$. At the same time, abnormally high expression of RHBDD1 was also found in breast cancer tissue, which promoted breast cancer progression by regulating the levels of p-Akt and CDK2 proteins, and was related to

A

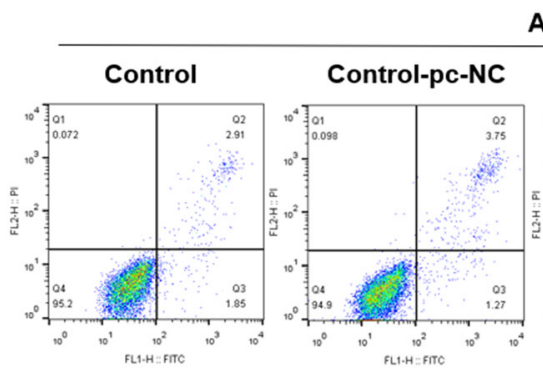

A549

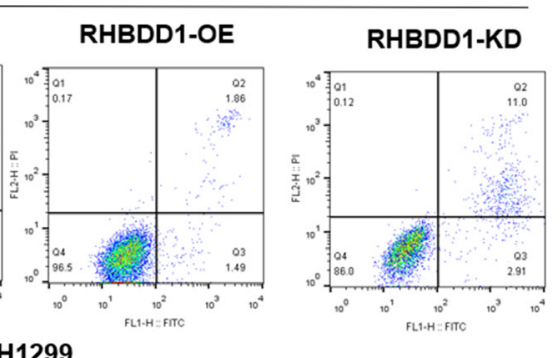

B
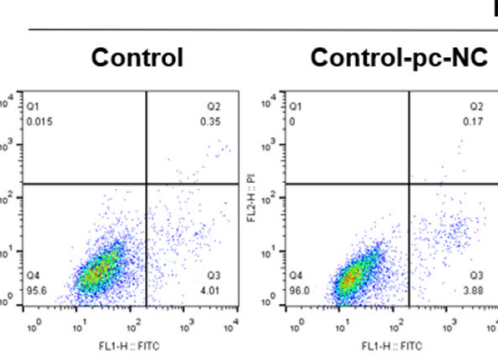

RHBDD1-OE
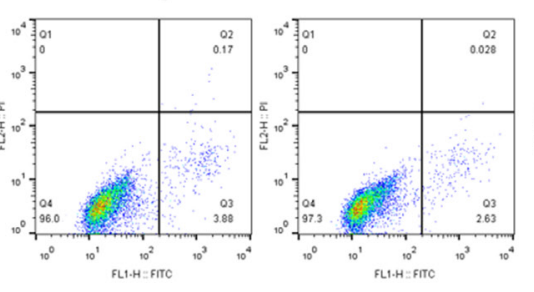

RHBDD1-KD
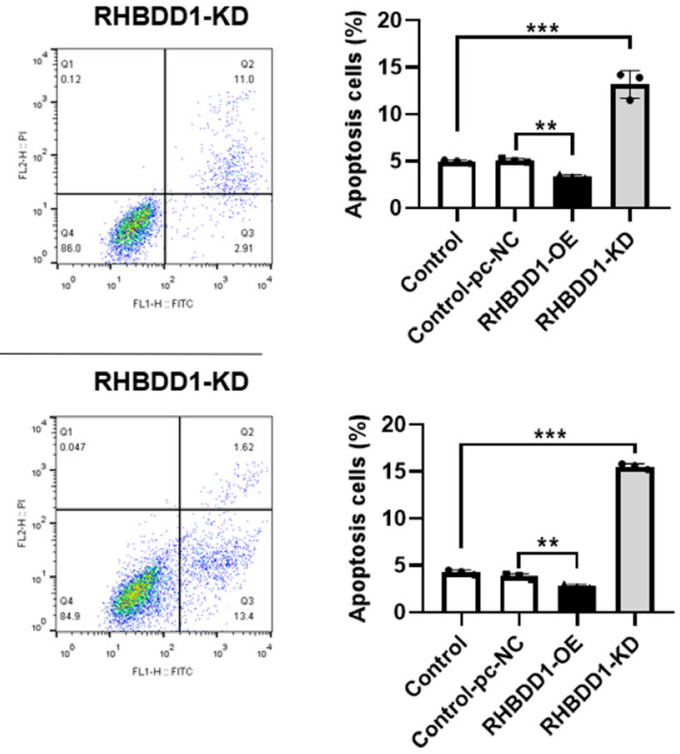

C
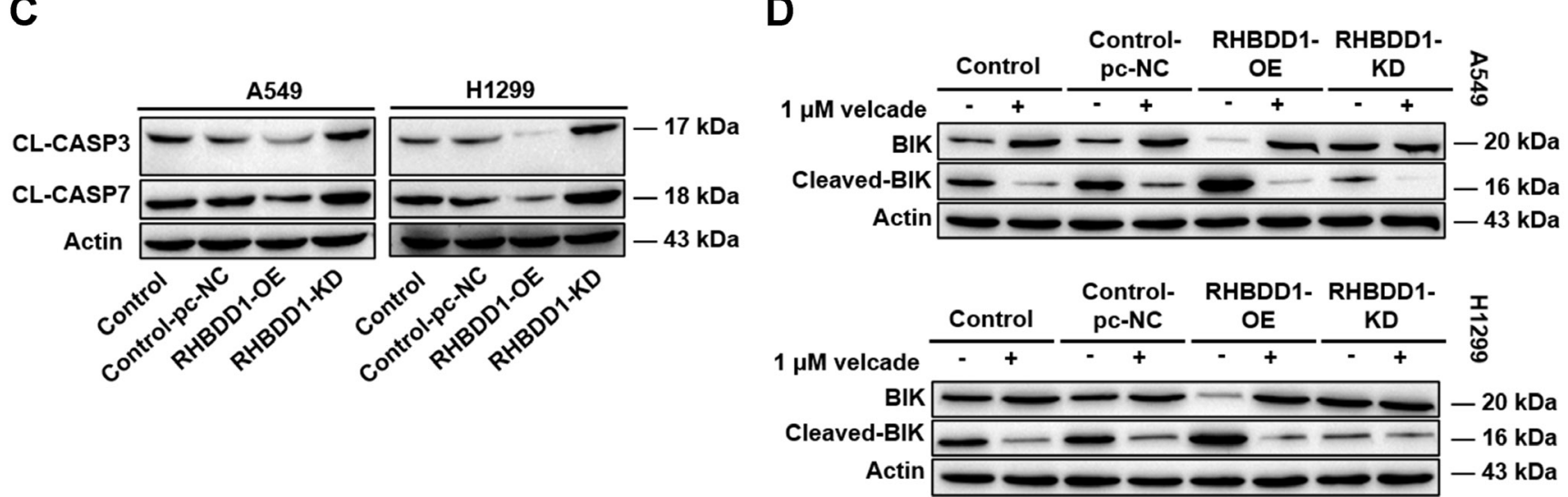

Figure 5. The expression level of RHBBD1 is inversely correlated with apoptosis. A, B) Flow cytometry was used to detect the percentage of apoptosis of each group. ${ }^{\star *} \mathrm{p}<0.01,{ }^{\star *}{ }^{*} \mathrm{p}<0.001$ vs. control or control-pc-NC. C) The protein levels of cleaved caspase 3 and cleaved caspase 7 were analyzed by western blotting, using $\beta$-actin as a loading control. The experiment was repeated three times. D) Western blotting was used to analyze the regulation of BIK by RHBDD1. 
the pathological tumor (pT) staging and estrogen receptor (ER)-positive breast cancer [8]. In ER-positive and triplenegative MDA-MB-231 cells, knocking out RHBDD1 can significantly downregulate cell proliferation and invasion [23]. In malignant gliomas, knocking down the high expression of RHBDD1 can also significantly inhibit the proliferation of cancer cells [25]. However, the mechanism by which RHBDD1 plays a role in NSCLC is still unclear. In this study, we observed that compared with normal lung bronchial epithelial cell line (HBE), RHBDD1 expression was higher in NSCLC cells. Previous studies have shown that RHBDD1 has different regulatory effects on the proliferation rate of tumor cells and non-tumor cells, which may be caused by the different expression levels of RHBDD1 in tumor and non-tumor cell lines [8]. We proposed that the promotive effect of RHBDD1 on the growth of NSCLC cells may be attributed to its regulatory effect on the cell cycle process and its influence on apoptosis.

In terms of RHBDD1 inhibiting apoptosis, a previous study has shown that the decrease of RHBDD1 could significantly increase breast cancer cell apoptosis [8]. Under different apoptotic stimuli, RHBDD1 activates and upregulates c-Jun and $\mathrm{Bcl}-2$, and finally inhibits the process of cell apoptosis [21]. In addition, as a protease, RHBDD1 can cleave the pro-apoptotic member BIK of the Bcl-2 family to inhibit cell apoptosis [22]. However, the regulatory mechanism of RHBDD1 in promoting apoptosis in NSCLC is still unclear. In our current study, we found that the knockdown of RHBDD1 significantly increased the apoptosis of NSCLC cells, while its overexpression reduced their apoptosis. In terms of molecular mechanism, we further researched and found that RHBDD1 in NSCLC cells could cleave BIK, resulting in upregulation of cleaved-BIK protein level. The addition of proteasome inhibitors can partially rescue the decrease in the proportion of apoptosis caused by RHBDD1 overexpression.

Our next step was to explore the effect of RHBDD1 on the cell cycle process of NSCLC and the possible molecular mechanism involved. In the RHBDD1 knockdown cells, we observed a significant increase in the proportion of cells in the G1 phase after 5 hours of TdR simultaneous treatment. Compared with the control groups, the absence of RHBDD1 caused the blockage of the progress from the G1 to the $S$ phase. Regarding the regulation of cycle-related proteins, we found that the decrease of RHBDD1 by siRNA significantly reduced the protein levels of CDK2 and p-Akt in A549 and H1299 cells. Conversely, the overexpression of RHBDD1 partially increased the protein levels of CDK2 and p-Akt. We speculated that RHBDD1, as a proteasome component, might participate in the proteolytic pathway of mediating cyclerelated proteins. As a result, we also proved that RHBDD1 promoted TGF- $\alpha$ maturation and release and further activated the EGFR-mediated Raf/MEK/ERK signaling pathway [10], resembling the findings found in colorectal cancer. In addition, RHBDD1 is well known for its role in ERAD (endoplasmic reticulum-associated degradation) and the rapid cell cycle of cancer cells causes their survival and growth to depend heavily on protein homeostasis [26, 27]. We supplementarily explored the possible relationship between RHBDD1 and ERAD in NSCLC, and the results were consistent with the previous findings that the rhomboid protease RHBDD1 residing in the endoplasmic reticulum was increased and cleaved the ubiquitinated ERAD substrate when the endoplasmic reticulum is stressed $[28,29]$.

Previous data also showed that the Akt-mediated signaling pathway was located downstream of EGFR, and a decrease in p-Akt level will downregulate the expression of CDK2 [30]. Therefore, we speculated that the absence of RHBDD1 may reduce the level of p-Akt through the Raf/ MEK/ERK signaling pathway, and ultimately inhibit the cell cycle process by downregulating the expression of CDK2.

In summary, RHBDD1 promotes NSCLC proliferation, inhibits the cell cycle process and apoptosis through the EGFR- and/or BIK-related signaling pathways, suggesting that RHBDD1 is a potential target for the treatment of NSCLC.

Supplementary information is available in the online version of the paper.

Acknowledgments: This study was supported by the Joint Foundation of Science \& Technology-Department of Yunnan Province and Kunming Medical University [Grant No.: 2019FE001(-115)]; The Foundation from Health Commission of Yunnan Province (Grant No.: 2018NS0271); and The Opening Project of the Clinical Medical Center of the First People's Hospital of Yunnan Province (Grant No.: 2021LCZXXF-HX11).

\section{References}

[1] BRAY F, FERLAY J, SOERJOMATARAM I, SIEGEL RL, TORRE LA et al. Global cancer statistics 2018: GLOBOCAN estimates of incidence and mortality worldwide for 36 cancers in 185 countries. CA Cancer J Clin 2018; 68: 394-424. https://doi.org/10.3322/caac.21492

[2] WAKELEE H, KELLY K, EDELMAN MJ. 50 Years of progress in the systemic therapy of non-small cell lung cancer. Am Soc Clin Oncol Educ Book 2014: 177-189. https://doi. org/10.14694/EdBook_AM.2014.34.177

[3] SCHABATH MB, COTE ML. Cancer Progress and Priorities: Lung Cancer. Cancer Epidemiol Biomarkers Prev 2019; 28: 1563-1579. https://doi.org/10.1158/1055-9965.Epi-19-0221

[4] SIEGEL RL, MILLER KD, JEMAL A. Cancer statistics, 2019. CA Cancer J Clin 2019; 69: 7-34. https://doi.org/10.3322/ caac. 21551

[5] FREEMAN M. Rhomboid proteases and their biological functions. Annu Rev Genet 2008; 42: 191-210. https://doi. org/10.1146/annurev.genet.42.110807.091628

[6] FREEMAN M. The rhomboid-like superfamily: molecular mechanisms and biological roles. Annu Rev Cell Dev Biol 2014; 30: 235-254. https://doi.org/10.1146/annurev-cellbio-100913-012944 
[7] ZHANG M, MIAO F, HUANG R, LIU W, ZHAO Y et al. RHBDD1 promotes colorectal cancer metastasis through the Wnt signaling pathway and its downstream target ZEB1. J Exp Clin Cancer Res 2018; 37: 22. https://doi.org/10.1186/ s13046-018-0687-5

[8] ZHANG X, ZHAO Y, WANG C, JU H, LIU W et al. Rhomboid domain-containing protein 1 promotes breast cancer progression by regulating the p-Akt and CDK2 levels. Cell Commun Signal 2018; 16: 65. https://doi.org/10.1186/ s12964-018-0267-5

[9] WUNDERLE L, KNOPF JD, KÜHNLE N, MORLÉ A, HEHN B et al. Rhomboid intramembrane protease $\mathrm{RH}$ BDL4 triggers ER-export and non-canonical secretion of membrane-anchored TGFa. Sci Rep 2016; 6: 27342. https:// doi.org/10.1038/srep27342

[10] SONG W, LIU W, ZHAO H, LI S, GUAN X et al. Rhomboid domain containing 1 promotes colorectal cancer growth through activation of the EGFR signalling pathway. Nat Commun 2015; 6: 8022. https://doi.org/10.1038/ncomms9022

[11] XU S, ZHANG H, WANG A, MA Y, GAN Y et al. Silibinin suppresses epithelial-mesenchymal transition in human non-small cell lung cancer cells by restraining RHBDD1. Cell Mol Biol Lett 2020; 25: 36. https://doi.org/10.1186/ s11658-020-00229-6

[12] PESCHON JJ, SLACK JL, REDDY P, STOCKING KL, SUNNARBORG SW et al. An essential role for ectodomain shedding in mammalian development. Science (New York, NY) 1998; 282: 1281-1284. https://doi.org/10.1126/science.282.5392.1281

[13] RIESE DJ, KIM ED, ELENIUS K, BUCKLEY S, KLAGSBRUN M et al. The epidermal growth factor receptor couples transforming growth factor-alpha, heparin-binding epidermal growth factor-like factor, and amphiregulin to $\mathrm{Neu}$, ErbB-3, and ErbB-4. J Biol Chem 1996; 271: 20047-20052. https://doi.org/10.1074/jbc.271.33.20047

[14] SANDGREN EP, LUETTEKE NC, PALMITER RD, BRINSTER RL, LEE DC. Overexpression of TGF alpha in transgenic mice: induction of epithelial hyperplasia, pancreatic metaplasia, and carcinoma of the breast. Cell 1990; 61: 11211135. https://doi.org/10.1016/0092-8674(90)90075-p

[15] JHAPPAN C, STAHLE C, HARKINS RN, FAUSTO N, SMITH GH et al. TGF alpha overexpression in transgenic mice induces liver neoplasia and abnormal development of the mammary gland and pancreas. Cell 1990; 61: 1137-1146. https://doi.org/10.1016/0092-8674(90)90076-q

[16] ZIOBER BL, WILLSON JK, HYMPHREY LE, CHILDRESSFIELDS K, BRATTAIN MG. Autocrine transforming growth factor-alpha is associated with progression of transformed properties in human colon cancer cells. J Biol Chem 1993; 268: 691-698.

[17] GRETEN FR, WAGNER M, WEBER CK, ZECHNER U, ADLER G et al. TGF alpha transgenic mice. A model of pancreatic cancer development. Pancreatology 2001; 1: 363-368. https://doi.org/10.1159/000055835
[18] PANDYA V, GITHAKA JM, PATEL N, VELDHOEN R HUGH J et al. BIK drives an aggressive breast cancer phenotype through sublethal apoptosis and predicts poor prognosis of ER-positive breast cancer. Cell Death Dis 2020; 11: 448. https://doi.org/10.1038/s41419-020-2654-2

[19] VAUX DL, CORY S, ADAMS JM. Bcl-2 gene promotes haemopoietic cell survival and cooperates with c-myc to immortalize pre-B cells. Nature 1988; 335: 440-442. https://doi. org/10.1038/335440a0

[20] PANDYA V, GLUBRECHT D, VOS L, HANSON J, DAMARAJU $S$ et al. The pro-apoptotic paradox: the $\mathrm{BH} 3$-only protein $\mathrm{Bcl}-2$ interacting killer (Bik) is prognostic for unfavorable outcomes in breast cancer. Oncotarget 2016; 7: 33272-33285. https://doi.org/10.18632/oncotarget.8924

[21] WANG Y, GUAN X, FOK KL, LI S, ZHANG X et al. A novel member of the Rhomboid family, RHBDD1, regulates BIKmediated apoptosis. Cell Mol Life Sci 2008; 65: 3822-3829. https://doi.org/10.1007/s00018-008-8452-0

[22] REN X, SONG W, LIU W, GUAN X, MIAO F et al. Rhomboid domain containing 1 inhibits cell apoptosis by upregulating AP-1 activity and its downstream target Bcl-3. FEBS Lett 2013; 587: 1793-1798. https://doi.org/10.1016/j.febslet.2013.04.033

[23] HUANG C, JI X, PENG Y, WU M, WU W et al. Silencing of rhomboid domain containing 1 to inhibit the metastasis of human breast cancer cells in vitro. Iran J Basic Med Sci 2018; 21: 1161-1166. https://doi.org/10.22038/ ijbms.2018.29788.7191

[24] PÉREZ-BENAVENTE B, FARRÀS R. Cell Synchronization Techniques to Study the Action of CDK Inhibitors. Methods Mol Biol (Clifton, NJ) 2016; 1336: 85-93. https://doi. org/10.1007/978-1-4939-2926-9_8

[25] WEI X, LV T, CHEN D, GUAN J. Lentiviral vector mediated delivery of RHBDD1 shRNA down regulated the proliferation of human glioblastoma cells. Technol Cancer Res Treat 2014; 13: 87-93. https://doi.org/10.7785/tcrt.2012.500362

[26] ANDERSON DJ, LE MOIGNE R, DJAKOVIC S, KUMAR B, RICE J et al. Targeting the AAA ATPase p97 as an Approach to Treat Cancer through Disruption of Protein Homeostasis. Cancer Cell 2015; 28: 653-665. https://doi.org/10.1016/j. ccell.2015.10.002

[27] VAN DRIE JH. Protein folding, protein homeostasis, and cancer. Chin J Cancer 2011; 30: 124-137. https://doi. org/10.5732/cjc.010.10162

[28] FLEIG L, BERGBOLD N, SAHASRABUDHE P, GEIGER $B$, KALTAK $L$ et al. Ubiquitin-dependent intramembrane rhomboid protease promotes ERAD of membrane proteins. Mol Cell 2012; 47: 558-569. https://doi.org/10.1016/j.molcel.2012.06.008

[29] GREENBLATT EJ, OLZMANN JA, KOPITO RR. Making the cut: intramembrane cleavage by a rhomboid protease promotes ERAD. Nat Struct Mol Biol 2012; 19: 979-981. https://doi.org/10.1038/nsmb.2398

[30] NAMIKI T, YAGUCHI T, NAKAMURA K, VALENCIA JC, COELHO SG et al. NUAK2 Amplification Coupled with PTEN Deficiency Promotes Melanoma Development via CDK Activation. Cancer Res 2015; 75: 2708-2715. https:// doi.org/10.1158/0008-5472.Can-13-3209 
https://doi.org/10.4149/neo_2021_210804N1107

\section{Rhomboid domain containing 1 promotes the growth of non-small cell lung cancer through the activation of EGFR and regulation of the BIK-mediated apoptosis}

Zhe-Yuan XU' ${ }^{1}$, Xu LI ${ }^{1}$, Rui-Hong YAO ${ }^{1}$, Li-Min YANG ${ }^{1}$, Hao PENG ${ }^{2}$, Wen-Jun REN ${ }^{1}$, Hong LU ${ }^{1}$, Ping WANG WA $^{1, *}$

Supplementary Information

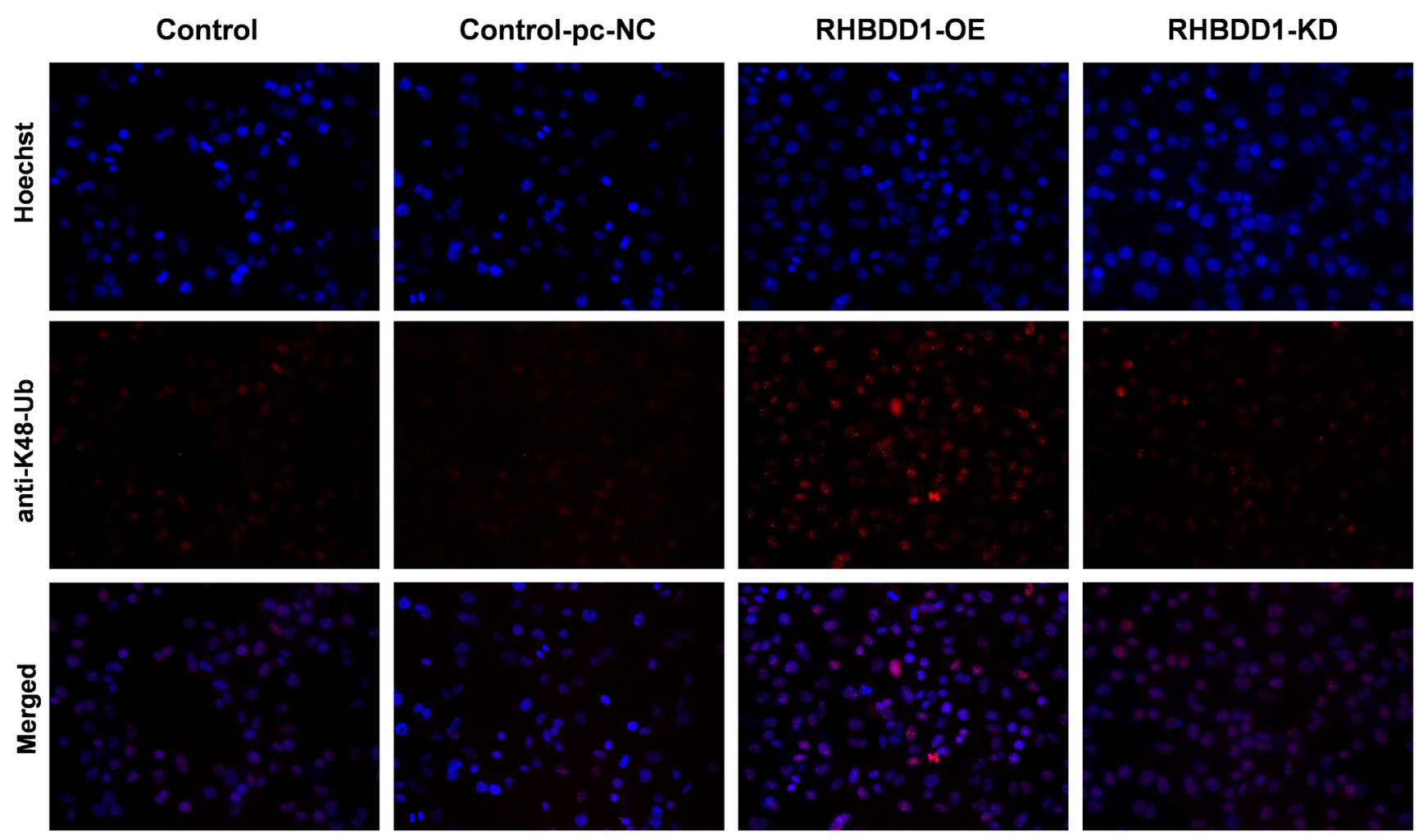

Supplementary Figure S1. RHBDD1 up-regulates the expression of K48-Ub and was positively correlated with ERAD. Immunofluorescence was employed to detect the expression location and level of K48 ubiquitin (K48-Ub) in each group. 